\title{
Efectos en la salud mental durante la pandemia
}

del coronavirus

Effects during the COVID-19 pandemic, in the mental health

Autor: Luisa Cristina Álvarez Ulloa

DOI: https://doi.org/10.19053/16923936.v18.n36.2020.12164

Para citar este artículo:

Ávarez Ulloa, L. C. (2020). Efectos en la salud mental durante la pandemia del coronavirus. Derecho y Realidad, 18, (36), 203-218.

Derecho y Realidad | ISSN Impreso 1692-3936 | ISSN En línea 2619-5607

Vol. 18 - Número 36 Julio - Diciembre de 2020 | pp. 203 - 218 DOI: https://doi.org/10.19053/16923936.v18.n36.2020.12164

(9)

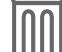




\section{EFECTOS EN LA SALUD MENTAL DURANTE LA PANDEMIA DEL CORONAVIRUS*}

Effects during the COVID-19 pandemic, in the mental health

Efeitos da pandemia COVID-19 sobre a saúde mental

\section{Luisa Cristina Álvarez Ulloa ${ }^{a}$}

luisa.alvarez@uptc.edu.co

Recepción: 31 de julio 2020

Aceptación: 19 de noviembre 2020

\section{RESUMEN}

El artículo a continuación tiene como objetivo establecer los efectos de la pandemia del COVID-19 en la salud mental a través del uso de una metodología mixta. Se plantea la posibilidad de que se incremente el desarrollo de enfermedades mentales, y con ello la crisis en los sistemas de la salud. Como resultado del análisis se estableció que las enfermedades mentales, como la depresión y la ansiedad, tendrán un aumento significativo durante la pandemia, y sus efectos continuarán muchas décadas después de que sea superada. Finalmente, se realizó una proyección de las consecuencias que, a largo plazo, generará la pandemia y se determinó que existe una alta probabilidad de que el $20 \%$ de la población mundial desarrolle algún tipo de enfermedad mental, como consecuencia del contexto generado por la pandemia.

\section{PALABRAS CLAVES}

Salud mental; COVID-19; pandemia; enfermedades mentales; crisis.

\begin{abstract}
The following article aims to establish the effects of the COVID-19 pandemic on mental health with a mixed methodology. It raises the possibility of increased development of mental illness, and with it the crisis in health systems. As a result of the analysis it was established that mental illnesses, such as depression and anxiety, will have a significant increase during the pandemic, and their effects will continue many decades after it is overcome. Finally, a projection of the long-term consequences of the pandemic was made, and it was determined that there is a high probability that $20 \%$ of the world's population will develop some form of mental illness as a result of the context generated by the pandemic.
\end{abstract}

\section{KEYWORDS}

Mental health; COVID-19; pandemic; mental illness; crisis.

\footnotetext{
* Artículo de reflexión.

a. Administradora Pública. Escuela Superior de Administración Pública.
} 


\section{RESUMO}

Este artigo tem como objetivo estabelecer os efeitos da pandemia COVID-19 sobre a saúde mental através do uso de uma metodologia mista. Surge a possibilidade de aumentar o desenvolvimento de doenças mentais e com isso a crise dos sistemas de saúde. Como resultado dessa análise, foi estabelecido que doenças mentais, como depressão e ansiedade, terão um aumento significativo durante a pandemia e seus efeitos continuarão muitas décadas após sua superação. Por fim, fez-se uma projeção das consequências que esta pandemia geraria a longo prazo e determinou-se que existe uma grande probabilidade de $20 \%$ da população mundial desenvolver algum tipo de doença mental, em consequência do contexto gerado pela pandemia.

\section{PALAVRAS-CHAVE}

Mental health; COVID-19; pandemic; doença mental; crise.

\section{INTRODUCCIÓN}

La salud mental es definida por la Organización Mundial de la Salud como un estado de bienestar, en el cual el individuo es consciente de sus propias capacidades, lo que le permite abordar las complejidades de la vida cotidiana. Sin embargo, las enfermedades de salud mental, definidas como trastornos psiquiátricos que impiden el desarrollo de las capacidades funcionales, con las que se aborda la vida cotidiana, se presentan como una de las grandes problemáticas a las que los gobiernos tendrán que dar respuesta durante el siglo. Ello se debe, en gran parte, al avance de una identidad construida desde la lógica de un sujeto individualista, propenso a la soledad, que el sistema capitalista fue afianzando a través del consumismo. Lo que ya se vislumbraba como uno de los grandes desafíos de la salud pública del siglo reciente, puede llegar a desatar una crisis de amplias magnitudes como consecuencia de la pandemia suscitada por el COVID-19. a la que se enfrenta la humanidad en el 2020. Ya que, por condiciones persistentes establecidas: el distanciamiento social, la cuarentena obligatoria y un contexto de crisis económica global creciente, se dibuja como el escenario perfecto para aumentar el porcentaje de personas que padecen enfermedades mentales e incrementar los casos de un número creciente de ciudadanos que ya padecían dichas enfermedades.

Por ello se busca, a partir de la aplicación de una metodología mixta, validar la hipótesis de que existirá un incremento de las enfermedades mentales como consecuencia del contexto creado por la pandemia del COVID-19. Para ello, en primer lugar se examinarán los precedentes históricos y científicos más relevantes, los cuales servirán como referentes en la interpretación de la primera catástrofe global a la que se enfrentan los sujetos. En segundo lugar, se presentarán los efectos psicológicos de las medidas implementadas para contener la pandemia, las cuales han tenido en diferentes segmentos de la población pertenecientes a varios países, se reportan desde el inicio del virus en diciembre de 2020 hasta mayo del 2020. Ello, con el objetivo de plantear una aproximación a las consecuencias que en el largo plazo dejará la emergencia en los seres humanos. En tercer lugar, a modo de conclusión, se formularán algunas sugerencias, que permitan anticiparse a través de soluciones certeras, a los desafíos que los sistemas de salud tendrán que enfrentar como consecuencia de los efectos de la crisis en la salud mental. Es importante dejar en claro que el presente abordaje cuenta con las limitaciones propias del contexto, debido a que la pandemia aún avanza por todas las regiones del mundo, y el virus reciente no cuenta con un abordaje científico amplio. Sin embargo, se tiene como objetivo impulsar a través del trabajo la inquietud, y curiosidad científica, que motive a más profesionales a contribuir desde todas las áreas de la ciencia, en el abordaje de la problemática que a todos atañe.

\section{PRECEDENTES HISTÓRICOS Y CIENTÍFICOS}

Los autores clásicos provenientes de la antigua Grecia son los primeros referentes que servirán para explorar las posibles consecuencias que, a nivel psicológico, 
podrían llegar a experimentar una gran parte de la población mundial, como resultado del momento histórico que enmarca la pandemia global. Desatada por el brote viral epidémico local desarrollado en la ciudad de Wuhan, China, cuya denominación taxonómica oficial, establecida por la Organización Mundial de la Salud, fue definida como COVID-19.

Observar el pasado, teniendo en cuenta que la historia reciente no vislumbro una situación de emergencia de una dimensión tan grande, es sin duda una estrategia acertada para observar la conducta humana frente a los desafíos que ha enfrentado. Lo que permitirá establecer los posibles traumas psicológicos que la crisis generó, especialmente en los países en vía de desarrollo donde los índices de pobreza y desigualdad son bastante evidentes. Lo que coloca un amplio segmento de la población en riesgo de desarrollar algún tipo de enfermedad mental durante el período.

Al remitirse a los grandes autores de la antigüedad se puede pensar en la Odisea y la Ilíada de Homero, una descripción precisa de la forma como los eruditos observaban el mundo y percibían las grandes tragedias. Si se observa la forma como Viktor Frankl, muchos siglos después, relataba su experiencia en un campo de concentración al finalizar la II guerra mundial, pueden encontrarse con detenimiento los grandes desafíos que la mente humana enfrenta en respuesta a los traumas, que dejan al pasar las desventuras que toman por sorpresa a los seres humanos, tal y como recuerda el gran Plutarco:

Ésta fue una ocasión en que se vio que una gran calamidad no sólo sirve para hacer prueba de los amigos, sino que también de los grandes generales, pues lo que antes de aquella batalla se graduaba en Fabio de cobardía e insensibilidad, después de ella pareció al punto, no ya una prudencia humana, sino un oráculo y providencia divina que prevé con anticipación aquellos sucesos que aun a los que los palpan se les hacen increíbles (Plutarco, 2017, pág. 268).
Para el sujeto moderno la dicotomía que deja en evidencia la primera pandemia de la postmodernidad, no sólo por la definición del sujeto actual en sí mismo, que incluye un entorno donde: "En la sociedad de consumidores nadie puede convertirse en sujeto, sin antes convertirse en producto. La característica más prominente de la sociedad de consumidores es su capacidad de transformar a los consumidores en productos consumibles" (Bauman, 2007, pág. 40). Sino que también, por el desarrollo de una identidad vinculada en algunos casos, principalmente a la lógica del consumismo, acompañada de dos ausencias: la primera cada vez más evidente de un propósito en sí mismo en la vida de algunos individuos y la segunda la de grandes generales o líderes capaces de anticiparse a esos sucesos que se palpan increíbles en el siglo, al igual que en la antigua Grecia.

Aproximadamente doce pandemias afectaron a la humanidad durante los siglos XIX y XX. Sin embargo, la más catastrófica fue la pandemia de gripe española. Ella se expandió por el mundo durante 1918 y 1919, "causando enfermedades agudas entre el 25 y 30 por ciento de la población mundial, produciendo la muerte a 40 millones de personas y convirtiéndose en el ancestro común de los virus de la gripe H1N1" (Taubenberger, 2009, pág. 88). Resulta importante hacer referencia a esa pandemia ya que permite entender, y predecir, el contexto al que por primera vez se enfrenta la humanidad posmoderna. Para el caso de la pandemia desatada por el COVID-19, reportó en mayo del 2020, según los últimos datos proporcionados por el Centro para el Control y la Prevención de Enfermedades: "un total de 4.786 .672 casos confirmados, $1,776,641$ de casos recuperados y un total de 317.695 fallecidos" (Centers for Disease Control and Prevetion, 2020). Cifras que presentan una tendencia que va en aumento, en espera de que se alcancen los picos más altos de casos reportados en América Latina, "donde el mayor número de casos para mayo del 2020 pertenece a Brasil con un número de 254.220 casos confirmados y un total de 16.792 fallecidos" (Ministério da Saúde, 
2020). También se espera un pico en la curva de casos en África y algunos brotes en Asia.

Los efectos psicológicos de la pandemia desatada por el virus de gripe española permiten indagar en una aproximación a los posibles efectos que el COVID-19 genere en la población mundial. A pesar de que la relación entre los efectos de la pandemia de 1918 y la salud mental, a largo plazo, no han sido estudiados con gran rigor en comparación con otros efectos de la pandemia, es importante analizar los resultados de algunas investigaciones sobre este hecho. Específicamente el estudio realizado por Mamelund, quien logró determinar, a través de su análisis científico, una conexión entre la gripe española y un aumento de las enfermedades neurológicas. Según su análisis: "Los sobrevivientes de la gripe española, informaron que tenían problemas para dormir, depresiones, distracciones mentales, presión arterial baja, mareos, complicaciones para enfrentar el trabajo y la vida cotidiana, semanas, meses o incluso años después de 1918-19" (Mamelund, 2003, pág. 5). Por otro lado, en el caso de los hospitales noruegos se informó que hubo un aumento: "en el número de pacientes hospitalizados por primera vez con trastornos mentales atribuidos a la gripe, reportando el incremento en un factor anual promedio de 7,2 en los 6 años siguientes a la pandemia" (Mamelund, 2003).

En el caso de los suicidios como consecuencia de la gripe española, pudo establecerse que: "las tasas de mortalidad por gripe en los Estados Unidos, durante los años 1918-1920 se relacionan significativamente con el suicidio, independientemente de factores dados como el consumo de alcohol y la cantidad de damnificados por la I guerra mundial" (Van Hartesveldt, 1992). Dicho vínculo entre el número de suicidios en Estados Unidos y la gripe española sería consecuencia de la disminución en la interacción social, devenida de las medidas establecidas para establecer el distanciamiento social, de la misma forma que se hace en el siglo XXI. Las medidas en aquel entonces para controlar la pandemia fueron muy similares, por ejemplo: se estableció el cierre de las escuelas y las prohibiciones de reuniones en lugares públicos. Además, la población se encontraba continuamente expuesta a una sensación de miedo, ya que los infectados podrían morir en tres días o perder en el proceso a sus seres queridos. Por otro lado, según el estudio realizado en Noruega, se estima que: "El número de personas, que sufrieron problemas mentales después de la gripe española, es mucho mayor de lo que el cálculo muestra ya que es probable que las personas afectadas por una melancolía postgripe, no visitaran al psiquiatra" (Mamelund, 2003, pág. 5).

Es evidente, que el contexto histórico al que se enfrentaban los seres humanos era distinto en aquel entonces, cuando apareció la pandemia de la gripe española, sobre todo por los desafíos planteados por la última etapa de la I guerra mundial, que dejaría graves consecuencias económicas especialmente para los países de Europa y algunos países de Asia. Sin embargo, los retos derivados, a nivel psicológico, son muy similares a los que enfrentan los ciudadanos del siglo XXI como consecuencia del COVID-19. A pesar de que el avance tecnológico es mayor que en aquel entonces, y la globalización facilita el intercambio de la información constantemente, lo cierto es que a nivel científico los retos y medidas con las que se enfrenta la pandemia son muy similares a las dadas en aquel entonces.

La pandemia a la que se enfrenta la humanidad da paso a una crisis económica global sin precedentes, en el siglo XXI, que impactará directamente en la salud mental de las personas. El Fondo Monetario Internacional estima a nivel mundial una caída de la economía, así lo informó el organismo en abril de 2020: "se proyecta que el crecimiento mundial descenderá inevitablemente $-3 \%$ en 2020. Se trata de un recorte de 6,3 puntos porcentuales con respecto a enero de 2020, una revisión importante en un período muy corto" (International Monetary Fund., 2020, pág. 1). Mientras que, según los datos que proporciona la CEPAL: "La pandemia del COVID-19, llevará a la mayor contracción 
económica en la historia de la región latinoamericana que caerá en un $-5,3 \%$ en el 2020, así mismo prevé un fuerte aumento del desempleo con efectos negativos en la pobreza y la desigualdad" (CEPAL, 2020, pág. 1). Ello permite entrever que muchas más personas perderán sus empleos, y con ello, en muchos casos, caerán en la pobreza. Complicando el contexto al que se enfrentan los seres humanos en todos los hemisferios, en donde no solo se ve amenazada su vida diariamente, por lo que los gobiernos decidieron llamar el enemigo invisible haciendo referencia al COVID-19, sino también el sustento de sus hogares, sus empleos, la rutina construida. Asimismo, se observan muchos proyectos truncados, frente a una respuesta certera ausente tanto de los líderes globales, como de un sistema sanitario no preparado para afrontar la emergencia, razón por la cual se ha visto colapsado.

Tal contexto adverso, en todas las esferas de la vida social, implica un desafío para gran parte de la población mundial. No solo por las condiciones socio-económicas que la pandemia ha generado, situando a las grandes potencias mundiales en una escalada de crisis mucho mayor a la enfrentada durante la Gran Depresión del 29', como se puede ver en los Estados Unidos, "donde alrededor de 36,5 millones de personas ya solicitaron el subsidio de desempleo" (U.S. Department of Labor, 2020). Sino que, a su vez, plantea el desafío de los sistemas de salud frente a las enfermedades mentales, que inevitablemente irán aumentando consecuencia de la pandemia y que impondrá recaídas en los segmentos de población que ya las padecían.

\section{Efectos psicológicos}

La salud mental es, según la OMS, un estado de bienestar en el cual el individuo es consciente de sus propias capacidades. Por lo que cabe resaltar: "lo que se refiere al núcleo de lo que nos hace humanos: la forma en que interactuamos, conectamos, aprendemos, trabajamos y experimentamos sufrimiento y felicidad, una buena salud mental apoya la capacidad de las personas para mostrar un comportamiento saludable" (The United
Nations, 2020, pág. 5). Por ello, resulta imperativo analizar la forma como la sociedad es afectada, a nivel psicológico, por la crisis. Ya que no se podría enfrentar una pandemia de estas dimensiones sin intervención y acompañamiento adecuado a través del monitoreo de la salud mental de todos los ciudadanos. Antes de que la pandemia fuera declarada, los informes que reportaban las estadísticas sobre las condiciones de salud mental ya eran preocupantes, incluidos los trastornos neurológicos producidos por el consumo de sustancias, el riesgo de suicidios y las discapacidades psicosociales derivadas. Según los datos proporcionados por la ONU la depresión: "Afecta a 264 millones de personas en el mundo, alrededor de la mitad de todas las enfermedades de salud mental comienzan a los 14 años y el suicidio es la segunda causa de muerte en jóvenes de 15 a 29 años" (The United Nations, 2020, pág. 5).

Para el caso de las personas que viven en entornos afectados por conflictos, la ONU reportaba que más de 1 de cada 5 personas tenían una condición poco favorable de salud mental. Si se considera la situación creada por la crisis actual como un periodo de conflicto, que afecta a la humanidad en su totalidad, el contexto se presenta como un entorno mucho más complicado que el derivado de las guerras y más exigente, en la medida de la resiliencia humana, por tener que ser enfrentado de forma distante y casi que solitaria. Se podría inferir por lo tanto, aumentando el tamaño de la muestra de la información proporcionada por la ONU, dado que toda la población se encontraría en riesgo de contraer el virus y aplicando la ley de probabilidad de Laplace, que un 20 $\%$ de la población mundial podría llegar a desarrollar una enfermedad mental como consecuencia de la crisis.

En un estudio reciente, realizado en Inglaterra por la University College de Londres, se encuestaron a un total de 74.000 personas con el objetivo de determinar cómo se sentían los adultos respecto a la situación de encierro, los consejos del gobierno, los sentimientos de soledad y bienestar. Ello, con el fin de determinar la forma cómo repercutían en la salud mental 
de los participantes, lo que expresaron los encuestados fue lo siguiente: "Una de cada cinco personas informaron que el Covid-19 les ha causado un gran estrés durante la última semana, en comparación con una de cada tres personas que manifestaron un gran estrés en los días previos a la cuarentena " (University College of London, 2020). Tal reporte estaría indicando que, en el caso de los ciudadanos ingleses, la implementación de las medidas de confinamiento impactaría, aparentemente, en una disminución del nivel de estrés de los encuestados. Sin embargo, si se calcula sobre la ley de probabilidad de Laplace, se puede inferir que la probabilidad de que una persona tenga estrés fuera de la cuarentena es de $0,3 \%$, mientras que la probabilidad de que una persona tenga estrés dentro de la cuarentena es de 0,2\% en el contexto del COVID-19. Con lo que se intuye que la cuarentena genera un nivel de estrés alto, a pesar de que las personas se encuentran aisladas fuera de los factores de la vida cotidiana que pueden acelerar el estrés. No obstante, es importante dejar en claro que dicha investigación continúa llevándose a cabo en la actualidad, al igual que muchos otros estudios, que son requeridos con rapidez por parte de la comunidad científica.

Por otro lado, evitar el estrés generado por una pandemia se dificulta a causa de diversos aspectos que pueden desatar crisis y reflejarlas en los distintos grupos de la esfera social. Por ejemplo, en los miembros de los hogares y los profesionales que no interactuaban directamente atendiendo la emergencia, era posible estar libres del virus. Sin embargo, al estar expuestos a una cuarentena obligatoria algunas investigaciones indican que los sujetos corren el riesgo de: "padecer confusión o ira, tendencias emocionales que pueden ser explosivas cuando varios miembros del hogar la soportan simultáneamente durante semanas e incluso meses. La proximidad forzada es un factor de riesgo para la agresión y la violencia doméstica" (Van Bavel, 2020 , pág. 462), lo que podría ser el causante del aumento de la violencia intrafamiliar y de los feminicidios que en la mayoría de los países del mundo comienzan a reportarse con tendencia a un aumento significativo, en los casos en que la violencia es denunciada. No obstante, las predicciones para los casos no reportados no son alentadoras, ya que, el contexto seguramente impide que muchas víctimas de violencia huyan del entorno de maltrato o reportarlo a las autoridades.

\subsection{Efectos Segmentados}

Los indicadores considerablemente importantes para considerar, para poder evaluar el impacto psicológico generado por la pandemia del COVID-19, son variados pero en conjunto muy importantes. Es por eso que se analizarán en los párrafos siguientes las emociones y patrones de conducta de diferentes segmentos de la población frente a la crisis. Ello, con el fin de identificar las enfermedades mentales más frecuentes que podrían ir en aumento, así mismo, se tendrán en cuenta datos estadísticos globales que permitirán generar un análisis probabilístico más amplio, en el caso de la conducta de los profesionales de la salud, frente a las situaciones extremas que enfrentan diariamente durante su trabajo. Se tendrá en cuenta el contexto actual donde los sistemas de salud se han visto en gran medida colapsados, a tal punto que los insumos han sido escasos y los tratamientos precarios para dar respuesta a un virus que tomó por sorpresa a la humanidad. Lo que ejerce estrés y presión psicológica, mucho mayor en los profesionales de la salud que en los demás segmentos de la sociedad. Allí, es evidente que "los trabajadores sanitarios de primera línea y los socorristas han estado expuestos a numerosos factores estresantes y garantizar la salud mental de los trabajadores sanitarios es un factor crítico para mantener la preparación, la respuesta y la recuperación de Covid-19" (The United Nations, 2020, pág. 11).

$\mathrm{Al}$ ser un virus tan reciente es complicado encontrar estudios científicos que, con un muestreo amplio, logren identificar los efectos que en el corto y largo plazo tendrá la pandemia en la población a nivel psicológico. A pesar de eso, si se usan como referencia algunos de los artículos científicos mencionados en el ítem anterior, que informaban las consecuencias en la salud mental de la población durante la 
gripe española de 1918 y las estadísticas que se expondrán en los párrafos siguientes de la situación actual, se pueden empezar a visualizar algunas de las repercusiones más notables que, en materia de salud mental, pueden llegar a afectar a la población a nivel global.

Uno de los aspectos más desafiantes es el distanciamiento social que, frente a la pandemia, se convirtió en la principal estrategia para prevenir el contagio y lo que implica para la salud mental de los seres humanos, definidos en esencia desde el punto de vista filosófico como seres sociales. Además, del hecho de que es posible que no puedan despedirse de sus seres queridos si estos llegaran a fallecer como consecuencia del virus. En tal medida, y con el objetivo de determinar la forma en que el COVID-19 está afectando la salud mental de las personas, la ONU presentó un informe en mayo del 2020, en el cual reportó, además de que se han registrado niveles de síntomas de depresión $\mathrm{y}$ ansiedad superiores a los habituales en varios países, que según un estudio realizado en la región de Amhara, Etiopía : " existe una tasa de prevalencia estimada del 33\% de los síntomas compatibles con el trastorno depresivo, un aumento de 3 veces, en comparación con las estimaciones de Etiopía antes de la pandemia" (The United Nations, 2020, pág. 7). Mientras que las estadísticas de Canadá reportan que: "el $20 \%$ de la población de entre 15 a 49 años aumentó el consumo de alcohol durante la pandemia" (The United Nations, 2020, pág. 7).

Lo que esos datos permiten determinar es que el aumento de una enfermedad, como el trastorno depresivo, en un país como Etiopía, cuyas características socioeconómicas son similares a las de los países en vía de desarrollo, crea la posibilidad de que exista un aumento del trastorno como consecuencia directa de la pandemia. En los países en vía de desarrollo con características similares, el aumento del consumo de alcohol en un país desarrollado como Canadá establece un referente sobre el que se puede calcular un posible aumento en el consumo de drogas legales, en los países desarrollados, como consecuencia de esta emergencia. Lo que agravaría la situación de algunos ciudadanos en países como Estados Unidos que, ya antes de esta crisis, presentaban un aumento en el consumo de opioides, con un porcentaje de mortalidad bastante alto: "en 2018, ocurrieron más de 31.000 muertes en Estados Unidos relacionadas con opioides sintéticos (excepto la metadona), reportaron un incremento del $10 \%$ de 2017 a 2018 y representaron el $67 \%$ de las muertes de pacientes implicados en opioides en 2018" (Centers for Disease and Control and Prevention, 2018). Mientras que en Australia: "150.000 personas dependen de opioides recetados, no obstante el caso del acceso ilícito a opioides (heroína) se dificulta, contribuyendo a la fabricación de opioides sintéticos que son altamente letales, lo que podría desatar una crisis sanitaria" (Suzzane, 2020).

Por otro lado, es importante resaltar que el alcohol es considerado una de las drogas legales más adictivas del mundo, ampliamente disponible en la actualidad: "se sabe que el alcohol tiene una multitud de efectos dañinos sobre el cerebro humano, siendo capaz de aumentar los niveles de dopamina entre un $40 \%$ y un $360 \%$, alrededor de un $22 \%$ de los consumidores de alcohol acabaran desarrollando una dependencia a él" (Nutt David, 2007). Por supuesto que, a medida que aumentan las cargas económicas y sociales, es posible empezar a proyectar un aumento en el desarrollo de diferentes enfermedades mentales, adicciones y distracciones en personas que no padecían ninguna, así como una recaída en los seres que ya padecían alguna de estas patologías.

Desde el punto de vista neurológico, algunos científicos comienzan a identificar el impacto del COVID-19 en el cerebro en base a estudios de necropsia que han demostrado: "la presencia de edema cerebral y degeneración neuronal en pacientes fallecidos por este virus, por otro lado se ha establecido que muchos pacientes presentan síntomas neurológicos inespecíficos y posiblemente de carácter sistémico, encefalopatía, encefalitis, y complicaciones cerebrovasculares" (Carod-Artal, 2020, pág. 
313). Ello demostraría que el virus tiene un impacto neurológico en el cerebro, por lo que sería necesario realizar el seguimiento pertinente de los pacientes que hayan superado la etapa crítica del virus. Con el fin de poder determinar si algunos presentan complicaciones de salud mental, en el largo plazo, como sucedió con algunos pacientes de la gripe española.

\subsection{Efectos en los profesionales de la salud}

Uno de los indicadores más relevantes que impacta directamente en la salud de la población es el estrés. En el caso de los profesionales de la salud es mucho mayor debido al alto riesgo de contagio que presentaron por su labor. Asimismo, sufrieron condiciones de trabajo precarias, estigmatización por parte de algunos segmentos de la sociedad, presión por la ausencia de una cura frente a un virus que llega a ser letal para varios pacientes y, en muchos casos, decisiones difíciles sobre la vida de los pacientes. Todas esas condiciones crean el panorama perfecto para que la salud mental de los profesionales de la salud se vea afectada en gran medida. Según el resultado de varias investigaciones realizadas sobre el impacto en la salud mental de los trabajadores sanitarios, pudo establecerse que en Canadá: "el 47\% de los trabajadores sanitarios informaron que necesitaban apoyo psicológico, además asociaron sentimientos negativos a su situación actual, el $67 \%$ dijeron sentirse ansiosos, inseguros $49 \%$, abrumados $40 \%$, indefensos $29 \%$, privados de sueño $28 \%$ y desalentados $28 \%$ " (Potloc Consumer Research, 2020). Mientras que en un estudio realizado en China, entre el 29 de enero y el 3 de febrero de 2020, los trabajadores de la salud reportaron: "altas tasas de depresión 50\%, ansiedad 45\%, insomnio 34\% y angustia 72\%" (Lai, y otros, 2020). Lo que permite inferir, en base a estos datos, que la ansiedad y la depresión son los trastornos más comunes que afectan a los profesionales de la salud en estos países.

Sin embargo, es preocupante el alto porcentaje de profesionales sanitarios que reportan algún síntoma. Especialmente si se considera como un factor determinante en el aumento de las tasas de suicidios, que ya era una preocupación durante el 2019, según algunas investigaciones llevadas a cabo en Estados Unidos: "Los médicos varones tienen tasas de suicidio hasta un $40 \%$ más altas que la población general, y las mujeres doctoras hasta un 130\% más altas" (Kalmoe, 2019, pág. 213). Como consecuencia de la pandemia, en varios países, los suicidios de trabajadores sanitarios comienzan a presentar un incremento, especialmente en los países donde el sistema sanitario ya ha colapsado. Es altamente probable que dicha cifra continúe en aumento al terminar la pandemia, similar al fenómeno presentado después de la peste española.

\subsection{Efectos en los niños, adolescentes y jóvenes}

El desarrollo adecuado de la salud mental, durante la niñez y la adolescencia, es fundamental como base de una vida plena durante la vida adulta. Más importante aún, es determinante en la prevención de enfermedades mentales en el futuro., Por ello resulta fundamental proveer un ambiente sano, de autoconfianza, comprensión y comunicación, que permitan el proceso apropiado de la primera infancia y adolescencia, ya que: "muchos problemas de salud mental, tienden a aparecer al final de la infancia o al comienzo de la adolescencia" (Organización Mundial de La Salud, 2020). Es por eso que se debe prestar especial atención en la forma como niños, adolescentes y jóvenes reaccionan a las condiciones de vida creadas por la pandemia, ya que podrían llegar a desarrollar trastornos de ansiedad y depresión.

La realidad es que el contexto actual del confinamiento ha colocado una gran presión en los hogares, lo que en gran medida ha contribuido a que surjan episodios de violencia en el entorno familiar, de los que los niños son testigos y algunas veces víctimas: "el $60 \%$ de todos los niños en todo el mundo viven en países donde existe un bloqueo total o parcial" (United Nations, 2020, pág. 10). Tal como se mencionó en párrafos anteriores, el confinamiento obligatorio generalmente produce niveles de estrés tan altos que proyectan el escenario perfecto para que 
aparezcan conductas agresivas. Los daños en la salud mental de los niños, durante el aislamiento social como consecuencia del COVID-19, pueden surgir de factores de riesgo tales como la desnutrición, la ausencia de estimulación y prolongadas situaciones de estrés crónico, que en el largo plazo dejaran secuelas irreversibles en los niños, a tal punto que: "son propensos a desarrollar desafíos de por vida a medida que su desarrollo neurológico se ve afectado, los niños que experimentan averías familiares durante este período de mayor estrés corren el riesgo de perder la sensación de apoyo y seguridad de la que depende su bienestar." (United Nations, 2020, pág. 12). Lo que a mediano plazo crearía una posible crisis en el sistema de salud pública, como consecuencia del aumento de los casos de niños, que años después de la pandemia, se habrán convertido en jóvenes con una alta probabilidad de padecer alguna enfermedad mental.

Durante la epidemia desatada por el virus del Ébola en África, algunas investigaciones reportaron que en Sierra Leona: "el número de niños diagnosticados con desnutrición aguda grave aumentó del 1,5 por ciento antes del brote, al 3,5 por ciento después del brote" (Barden-O'Fallon, 2015, pág. 5). Consecuencia directa de la crisis económica generada por la epidemia. Por otro lado, es importante mencionar que existe una alta probabilidad de que dichos niños desarrollen enfermedades mentales en su juventud o vida adulta, ya que la nutrición en la primera infancia es un componente primordial del desarrollo neurológico.

En el caso de los adolescentes y los jóvenes, es importante mencionar el hecho de que el contexto de la pandemia del COVID-19 plantea grandes desafíos, basados en el hecho de que tienen que enfrentar el cierre de las escuelas y las universidades. Con una alternativa de educación digital a la que lamentablemente no todos pueden acceder por falta de recursos, especialmente en las economías de los países pobres. La dificultad de poder tener interacciones sociales constantes en lugares públicos, sumados a las dificultades económicas que empiezan a atravesar los hogares a nivel global, son una combinación perfecta que puede estar exponiendo a los adolescentes y jóvenes a altos niveles de estrés. Combinados con la etapa que atraviesan puede ser peligrosa y en muchos casos letal, ya que puede incrementar la aparición de adicciones, trastornos de ansiedad y depresión, que en algunos casos no llegan a ser reportados. Debido a que muchos adolescentes y jóvenes encuentran dificultades para expresarse, o buscar ayuda, al momento de hablar de enfermedades mentales. Ello se puede atribuir, en gran parte, a los estigmas que encuentran instaurados en la sociedad respecto a las patologías que pueden derivar de complicaciones con la salud mental.

Un estudio realizado a jóvenes con necesidades de salud mental en el Reino Unido, determinó que: "el $32 \%$ de los participantes estuvo de acuerdo en que la pandemia había empeorado mucho su salud mental" (YoungMinds, 2020, pág. 3). Sin embargo, tales tendencias, en el reporte del aumento de trastornos por enfermedades mentales, seguramente se incrementen a medida que el COVID-19 siga expandiéndose.

\subsection{Efectos en los adultos mayores}

Los adultos mayores son uno de los segmentos de población en mayor riesgo, en el nivel de letalidad, frente al COVID-19. Situación por la cual, se les considera vulnerables frente al virus, a tal punto que en algunos países se les han impuesto restricciones específicas a las personas sobre cierto rango de edad, con el fin de facilitar su distanciamiento social. Es un hecho que la población mundial está envejeciendo de manera acelerada, según proyecto la OMS: "Entre 2015 y 2050 la proporción de la población mundial mayor de 60 años se multiplicará casi por dos pasando del 12\% al 22\%" (Organización Mundial de la Salud, 2017). Enfrentando los desafíos que la edad adulta trae por sí sola, factores como la jubilación, la reducción de los ingresos económicos, experiencias continuas de dolor derivadas de problemas de salud propios de la edad o la pérdida de seres queridos, pueden generar depresión y soledad que, en el caso de los adultos mayores que padecen 
discapacidades, puede ser mucho mayor. A tales circunstancias se les suman los desafíos que la pandemia del COVID-19 deja a su paso, estableciendo cuarentenas por largos períodos de tiempo en la mayoría de los países y fomentando el aislamiento social, que en el caso de los adultos mayores puede generar un incremento significativo en los padecimientos por enfermedades que atacan la salud mental.

Enfermedades como la demencia, síndrome caracterizado por la reducción de la memoria y la capacidad de pensar, proyectaban un incremento probable en los próximos años. Según datos reportados por la OMS, se calcula que en el mundo: "Hay unos 47,5 millones de personas aquejadas de demencia. Se prevé que el número de estas personas aumentará a 75,6 millones en 2030 y a 135,5 millones en 2050; la mayoría de esos pacientes vivirán en países de ingresos bajos" (Organización Mundial de la Salud, 2017). Por otro lado, en el caso de la depresión en mayorea de 60 años, se preveía un incremento antes de la pandemia: "La depresión unipolar afecta a un 7\% de la población de ancianos en general y representa un $5,7 \%$ de los años vividos con una discapacidad entre las personas de 60 años y mayores" (Organización Mundial de la Salud, 2017). Teniendo en cuenta que el aumento estimado en la población de adultos mayores a nivel mundial es de 900 millones a 2000 millones de personas mayores de 60 años, puede estimarse que los efectos en la salud mental de este grupo etario aumenten significativamente con el desarrollo de enfermedades mentales, consecuencia del contexto socioeconómico derivado de esta crisis. Sobre todo, si se tiene en cuenta que las condiciones dadas favorecen el aislamiento de los adultos mayores y los hacen más vulnerables a factores de riesgo como el maltrato en todas sus dimensiones: físico, psicológico, económico. Asimismo, son más susceptibles al abandono y a la violencia, que pueden llegar a propiciar el desarrollo de varias enfermedades mentales entre ellas demencia y depresión.

Uno de los aspectos más preocupantes que la pandemia puso en evidencia es, sin duda, el hecho de que "las personas mayores están siendo abandonadas en hogares de cuidado y la cantidad de cadáveres encontrados en residencias de ancianos son alarmantes" (United Nations, 2020). Es por ello que son uno de los segmentos de población que se encuentran en mayor riesgo de padecer alguna enfermedad mental como consecuencia de la pandemia. Tanto por el estrés al que se enfrentan cotidianamente, como por la ausencia de una garantía plena de sus derechos. Tal y como lo expresó uno de los expertos delegados de las Naciones Unidas: "Preocupa que la decisión en la asignación de recursos médicos escasos, como los ventiladores en las unidades de cuidados intensivos, puedan tomarse únicamente sobre la base de la edad, negando a las personas mayores su derecho a la vida" (United Nations, 2020). Ya que, como lo han expresado los profesionales de la salud, son continuadas las ocasiones en las que han tenido que tomar decisiones tan importantes como a quien salvarle la vida, basadas en el criterio de la edad de los pacientes.

\subsection{Efectos en las Mujeres}

Las mujeres son un segmento de la población expuesto a situaciones de violencia constante, que en muchos de los casos terminan convirtiéndolas en víctimas de feminicidios. La salud mental de las mujeres, en el período de pandemia, presenta factores de riesgo generados por la vulnerabilidad económica, el estrés y la incertidumbre de situación de crisis. En el caso de las mujeres que están en período avanzado de gestación, es probable que experimenten ansiedad y en el caso de las mujeres diagnosticadas con COVID-19 que están embarazadas o ya son madres lactantes, las emociones de miedo y depresión por no poder tener contacto físico con el bebé, serán, sin ninguna duda, constantes. Según los datos proporcionados por las investigaciones llevadas a cabo por UNFPA, puede proyectarse que: " 47 millones de mujeres en 114 países de ingresos bajos y medianos, no puedan acceder a anticonceptivos y que se produzcan 7 millones de embarazos no deseados, mientras que se puede esperar que se produzcan 31 millones de casos adicionales de violencia de género" (United 
Nations Population Fund, 2020). Dichas proyecciones efectuadas por el organismo se realizaron sobre la base de una cuarentena de seis meses, la UNFPA proyecta que la cifra aumentaría en dos millones en el caso de las mujeres sin acceso a los anticonceptivos, mientras que en los casos de violencia de género puede estimarse un incremento en esos tres meses adicionales de 15 millones.

Los niveles de estrés presentan un aumento significativo en las mujeres, según datos reportados por la ONU, en una encuesta realizada en la India durante la pandemia se indicó que: "el $66 \%$ de las mujeres informaron estar estresadas en comparación con el 34\% de los hombres" (The United Nations, 2020, pág. 13). Lo que podría derivar en el aumento de los casos de trastornos por depresión y un aumento de los niveles de estrés. Dentro de tal segmento de población, al igual que en los mencionados en párrafos anteriores, existen un sin número de circunstancias que hacen que las personas que enfrentan condiciones de precariedad y violencia sean más propensas a padecer enfermedades mentales. Otros segmentos de población, que son vulnerables a padecer dichas circunstancias, son los refugiados, las víctimas de desplazamiento forzado, que enfrentan condiciones de extrema pobreza, al igual que los segmentos sociales que no tienen acceso a los servicios básicos como agua potable.

Por otro lado, una de las patologías más complejas que podrían afectar a un gran porcentaje de la población a nivel global, en todos los segmentos de la población, es el trastorno de estrés postraumático. Generalmente atribuido a los veteranos de guerra, pero caracterizado específicamente por síntomas emocionales y físicos, que suelen aparecer después de la exposición a un acontecimiento traumático que, como el COVID-19, podría llegar a transformarse en una de las enfermedades mentales más comunes dentro de los próximos 20 años. Proyección que podría tener una alta probabilidad de ser certera, teniendo en cuenta los altos índices de estrés reportados en los resultados de las investigaciones científicas, mencionadas anteriormente, y la exposición constante de los sujetos a condiciones cada vez más inciertas y complejas.

\section{CONCLUSIONES}

Como resultado de la aplicación de la ley de probabilidad de Laplace, pudo determinarse que existe una alta probabilidad de que el $20 \%$ de la población mundial desarrolle algún tipo de enfermedad mental, como consecuencia del contexto generado por el COVID-19. Las enfermedades mentales que se proyecta aumentaran significativamente son la depresión y la ansiedad. En el caso de los países en vía de desarrollo, se estima en base a los datos proporcionados por Etiopia, que exista un aumento en el padecimiento del trastorno depresivo. Mientras que en el caso de los países desarrollados, se puede inferir tomando como base los datos proporcionados por Canadá, donde existe una alta probabilidad de aumento en el consumo de drogas legales e ilegales. Lo que puede, a largo plazo, contribuir con un aumento significativo de la población con adicciones y al avance de enfermedades mentales derivado del consumo de sustancias.

Aspectos como las complicaciones derivadas de las circunstancias socioeconómicas de la crisis impactan directamente en la salud mental de las personas. Las cuales, dado el contexto, son más vulnerables a padecer o intensificar adicciones, tener distracciones mentales derivadas de los altos niveles de estrés, desarrollar enfermedades mentales a pesar de no haber tenido ningún tipo de padecimiento antes o, en el caso de los segmentos de población con patologías, una alta probabilidad de que se intensifiquen. Aún no existe un conocimiento en profundidad de los efectos que el COVID-19 puede tener en pacientes recuperados. Sin embargo, será necesario realizar un monitoreo de los pacientes que se han recuperado, ya que se sabe que el virus tiene un impacto neurológico en el cerebro que aún está por determinarse.

Las conductas agresivas presentarán, además, un aumento significativo como 
consecuencia del aislamiento obligatorio, que suele favorecer la aparición de escenas de violencia cada vez más cotidianas. En el caso de los niños, debe realizarse un monitoreo constante a su salud mental, ya que en el entorno familiar son más vulnerables a sufrir violencia u observarla, por las circunstancias derivadas de la pandemia. Asimismo, se encuentran en un alto riesgo de padecer desnutrición, tener que enfrentar circunstancias de estrés crónico, y ausencia de la estimulación que requieren, sobre todo durante la primera infancia, lo que podría llegar a causar daños neurológicos y con ellos enfermedades mentales que afecten su desarrollo durante toda la vida adulta.

Es indispensable que los países comiencen a invertir en programas que sean asertivos en el manejo del incremento de la demanda, en el sector de la salud pública, por parte de las personas que desarrollen enfermedades mentales, como consecuencia del contexto catastrófico que ha dejado el COVID-19. Es evidente que se requieren soluciones precisas y contundentes que permitan prevenir una crisis generada por las enfermedades de salud mental, crear campañas de acompañamiento a través de distintos medios de comunicación y medios digitales que permitan a las personas aprender sobre el manejo de las emociones, es imperativo.

Los niveles de estrés a los son expuestos los profesionales sanitarios son tóxicos y demasiados peligrosos, podrían incrementar el número de suicidios, que para el 2019 ya era bastante alto en este grupo de profesionales. Es fundamental que la logística hospitalaria plantee estrategias que permitan brindar un acompañamiento completo y de calidad a los profesionales sanitarios durante esta etapa. El riesgo de que exista un incremento en la población, con padecimientos derivados del trastorno de ansiedad o depresión es muy alto. Por lo tanto, se requiere una intervención por parte de todas las instituciones que conforman los grupos sociales, se requiere de un trabajo en equipo que sincronice diversos aspectos de la esfera social en este tiempo de emergencia. Así, por ejemplo, desde las escuelas que en la mayoría de los países están operando de forma no presencial, plantear un acompañamiento por parte de los maestros, que permita a los estudiantes tener una contención desde la academia. En el caso de las universidades es muy importante que se faciliten las herramientas a todos los alumnos para tener acceso a las clases, en el hogar deben instaurarse espacios de diálogo que le permitan a los grupos familiares expresar sus emociones frente a la situación de confinamiento. Es importante tejer redes que conecten todas las instituciones del estado, incluyendo la creación de sistemas de alerta que permitan reportar los casos de abuso y de violencia.

Los efectos que en la salud mental impactarán en la humanidad entera muchas décadas después de que la pandemia sea superada, especialmente porque las secuelas de los traumas generados por esta situación trascenderán varias generaciones. Por ello es importante que la inversión e implementación de programas enfocados en la salud mental se incrementen lo antes posible. Evidentemente, dadas las cifras analizadas en este artículo, de no propiciarse es altamente probable que la sociedad enfrente una crisis de salud mental, que impactará negativamente en el crecimiento y desarrollo económico de los países. En el caso de las Naciones que ya sufrieron epidemias anteriores recientes, como es el caso del continente africano con el Ébola, es muy importante que se desarrollen investigaciones científicas que permitan establecer el estado de la salud mental de los ciudadanos. Ya que, como es bien sabido, la exposición continua a conflictos como los creados por esta pandemia tiende a crear en los individuos conductas cada vez más agresivas que facilitan la aparición de conductas violentas. No menos importante, es el hecho de que pueden llegar a incrementar los índices de padecimientos por el trastorno depresivo y de ansiedad.

La posibilidad de que el trastorno de estrés postraumático sea el trastorno psicológico más común en el escenario desafiante de la post-pandemia es altamente probable. En primer lugar, por los desafíos 
a los que se enfrentan los profesionales de la salud, quienes en su gran mayoría ya comienzan a comparar las unidades hospitalarias con un escenario bélico, en el que las decisiones tienen que tomarse rápidamente y los recursos son escasos. En segundo lugar, porque las consecuencias de los altos niveles de estrés reportados en diferentes lugares del mundo se verán más a largo plazo, debido a que las restricciones implantadas en algunos lugares como parte de las medidas para prevenir el contagio influyen en el comportamiento de las personas. Además de que han limitado el acceso al sistema sanitario por la necesidad de que este se encuentre disponible y alerta para atender la emergencia.

En muchos países del mundo se instauró un debate basado en la economía, reduciendo la cuestión a un asunto monetario que seguramente seguirá profundizando las desigualdades. Sin embargo, es muy probable que lo que deje a su paso la pandemia sea un cambio de Era. Que lleve a cuestionar al sujeto de la postmodernidad sobre el propósito de su existencia, un factor primordial en el desarrollo de la salud mental, que dota a la humanidad de sentido y a la sociedad de interrogantes, donde las grandes innovaciones nacen.

\section{REFERENCIAS BIBLIOGRÁFICAS}

» International Monetary Fund. (14 de Abril de 2020). International Monetary Fund. Obtenido de World Economic Outlook, April 2020: The Great Lockdown: https://www.imf.org/en/Publications/WEO/Issues/2020/04/14/weo-april2020\#Chapter 1

» Barden-O'Fallon, J. A. (4 de Agosto de 2015). US National Library of Medicine. Obtenido de Rapid Assessment of Ebola-Related Implications for Reproductive, Maternal, Newborn and Child Health Service Delivery and Utilization in Guinea: https://www.ncbi.nlm.nih.gov/pmc/articles/PMC4542265/

» Bauman, Z. (2007). Vida de Consumo. México: Fondo de Cultura Económica.

»Carod-Artal, F. (2020). Complicaciones neurológicas por coronavirus y COVID-19. Revista de Neurología, 70 (09): Recuperado 27 de mayo de 2020 de: https://www.neurologia.com/articulo/2020179

» Centers for Disease and Control and Prevention. (2018). Opioid Overdose. Obtenido de Synthetic Opioid Overdose Data: https://www.cdc.gov/drugoverdose/ data/fentanyl.html

»Centers for Disease Control and Prevetion. (18 de Mayo de 2020). Centers for Disease Control and Prevention. Recuperado 5 de junio de 2020 de: https://n9.cl/ $\mathrm{z7sj}$

$»$ CEPAL. (ABRIL de 2020). América Latina y el Caribe: Proyecciones de Crecimiento 2020 . Recuperado 6 de mayo de 2020 de: https://n9.cl/2inb1.

» Kalmoe, M. C. (junio de 2019). US National Library of Medicine, National Institutes of Health. Obtenido de Physician Suicide: A Call to Action, el 20 de agosto de 2020 de: https://www.ncbi.nlm.nih.gov/pmc/articles/PMC6690303/

» Lai, J., SimengMa, M., YingWang, M., ZhongxiangCai, M., JianboHu, M., NingWei, M., ShaohuaHu, M. (2 de Marzo de 2020). FactorsAssociated With Mental Health Outcomes Among HealthCare Workers Exposed to Coronavirus Disease 2019. Jama Network. file://C:/Users/Usuario/AppData/Local/Packages/ 
Microsoft.MicrosoftEdge_8wekyb3d8bbwe/TempState/Downloads/lai_2020_ oi_200192\%20(1).pdf

» Mamelund, S.-E. (Octubre de 2003). Effects of the Spanish Influenza Pandemic of 1918-19, on Later Life Mortality of Norwegian Cohorts Born. ResearchGate. Recuperado 06 de mayo de 2020 de: https://n9.cl/rkicp.

» Ministério da Saúde. (Mayo de 2020). Boletins Epidemiológicos. Recuperado 28 de junio de 2020 de: https://coronavirus.saude.gov.br/boletins-epidemiologicos

» Nutt David, K. L. (24 de Marzo de 2007). The Lancet . Development of a rational scale to assess the harm of drugs of potential misuse. Recuperado 30 de junio de 2020 de: https://www.thelancet.com/journals/lancet/article/PIIS01406736(07)60464-4/fulltext

» Organización Mundial de la Salud. (12 de Diciembre de 2017). La salud mental y los adultos mayores. Recuperado 05 de marzo de 2020 de: https://n9.cl/zqvzg.

» Organización Mundial de la Salud. (18 de Mayo de 2020). Salud de la madre, el recién nacido, del niño y del adolescente. Recuperado 05 de junio de 2020 de: https://n9.cl/kv4r.

» Plutarco. (2017). Vidas Paralelas. Menorca, España: Textos.Info.

» Potloc Consumer Research. (16 de Abril de 2020). Potloc Study: Canadian health workers share their insights from the front lines of the COVID-19 pandemic. Potloc. Recuperado 09 de junio de 2020 de: https://n9.cl/m8ep.

» Suzzane, N. (23 de Abril de 2020). Monash University. By the double: COVID-19, opioids, and the threat of a syndemic. Recuperado 03 de junio de 2020 de: https:// n9.cl/actss.

» Taubenberger, J. K. (3 de Agosto de 2009). US National Library of Medicine. The Origin and Virulence of the 1918 "Spanish" Influenza Virus. Recuperado 03 de abril de 2020 de: https://www.ncbi.nlm.nih.gov/pmc/articles/PMC2720273/

» The United Nations. (14 de Mayo de 2020). United Nations. Obtenido de COVID-19 and the Need for Action on Mental Health. Recuperado 24 de julio de 2020 de: https://n9.cl/91tnv.

» U.S. Department of Labor. (Abril de 2020). U.S. Department of Labor. Recuperado 9 de mayo de 2020 de U.S. Department of Labor: https://www.dol.gov/

» United Nations. (27 de Marzo de 2020). United Nations. Recuperado 09 de abril de 2020 de Department of Economic and Social Affairs: https://www.un.org/development/desa/ageing/news/2020/03/covid-19/

» United Nations. (15 de Abril de 2020). United Nations. Policy Brief: The Impact of COVID-19 On Children. Recuperado 30 de abril de 2020 de: https://n9.cl/ryko.

» United Nations Population Fund . (28 de Abril de 2020). United Nations Population Fund . Obtenido de New UNFPA projections predict calamitous impact on women's health as COVID-19 pandemic continues. Recuperado 30 de abril de 2020 de: https://n9.cl/61vo6.

» University College of London. (17 de April de 2020). UCL. Stress over Covid-19 keeping fewer people up at night. Recuperado 30 de abril de 2020 de: https:// n9.cl/0qc46.

» Van Bavel, J. (2020). Using social and behavioural science to support COVID-19 pandemic response. Nature, 7. 
» Van Hartesveldt, F. (1992). The 1918-1919 Pandemic Of Influenza: The Urban Impact In The Western World. New York: Edwin Mellen Press.

» YoungMinds. (Marzo de 2020). Coronavirus: Impact on young people with mental health need. Recuperado 15 de abril de 2020 de: https://n9.cl/iay5 